\title{
Automatic Multiple Tilt Series Acquisition in Leginon
}

\author{
G.J. Jensen*, C. Suloway*, Q.S. Zheng**, and D.A. Agard****** \\ * Division of Biology, California Institute of Technology, Pasadena, CA 91125 \\ ** The Howard Hughes Medical Institute, University of California, San Francisco, CA 94143-2240 \\ *** The W.M. Keck Advanced Microscopy Laboratory, Department of Biochemistry and \\ Biophysics, University of California, San Francisco, CA 94143-2240
}

A number of software packages are available that automate tilt series acquisition for electron tomography. Faced with an ever-increasing need to collect more tomograms, we implemented a version of the UCSFTomo [1] predictive tracking algorithm within Leginon [2], a popular software package originally designed to automate single-particle data collection on.

Additional features have been added to Leginon to facilitate automated tilt series collection, including a new Leginon tomography "node" and new tables added to the database for storing the tilt series, tomogram, and predictive tracking algorithm information. Leginon web tools have been enhanced with pages for viewing tomography data, including a utility for diagnosing tracking failures when they occur.

Data acquisition is started using Leginon's powerful grid atlas function, a low-dose survey montage of the central region of the grid accessible for dual-axis tilt series is produced. Areas of the atlas can then be selected in the user interface to be surveyed again into montages at increased magnifications. The user chooses a list of targets and priorities from the montages, and the grid coordinates are then computed. Leginon then proceeds from one target to the next performing all of the tasks necessary for collecting high quality cryo-EM tilt series data using an energy filter, including setting focus and eucentric height, realigning the energy filter slit, and recording full tilt series without ever needing human interaction. The current rate of data acquisition is roughly 30 seconds per tilt image on a $2 \mathrm{~K}$ by $2 \mathrm{~K}$ CCD camera, comparable to the original UCSFTomo. Targets are kept centered during the entire period of data collection by the Leginon drift monitoring system to ensure tilt series are taken at the correct locations on the specimen regardless of changes over time.

We expect this software will significantly increase productivity by facilitating routine 24-hour data collection; efficiently re-finding targets after grid rotation for collection of a second, dual-axis tilt series; reducing the perturbations caused by constantly rotating users and their individualized set-up routines, and identifying microscope performance problems immediately [3].

\section{References}

[1] Q.S. Zheng et al., J. Struct. Bio. 147 (2004) 91-101.

[2] C. Suloway et al., J. Struct. Bio. 151 (2005) 41-60.

[3] This research was supported in part by NIH grant P01 GM66521 to G.J.J., DOE grant DEFG02-04ER63785 to G.J.J., a Searle Scholar Award to G.J.J., and gifts to Caltech from the Ralph M. Parsons Foundation, the Agouron Institute, and the Gordon and Betty Moore Foundation. 


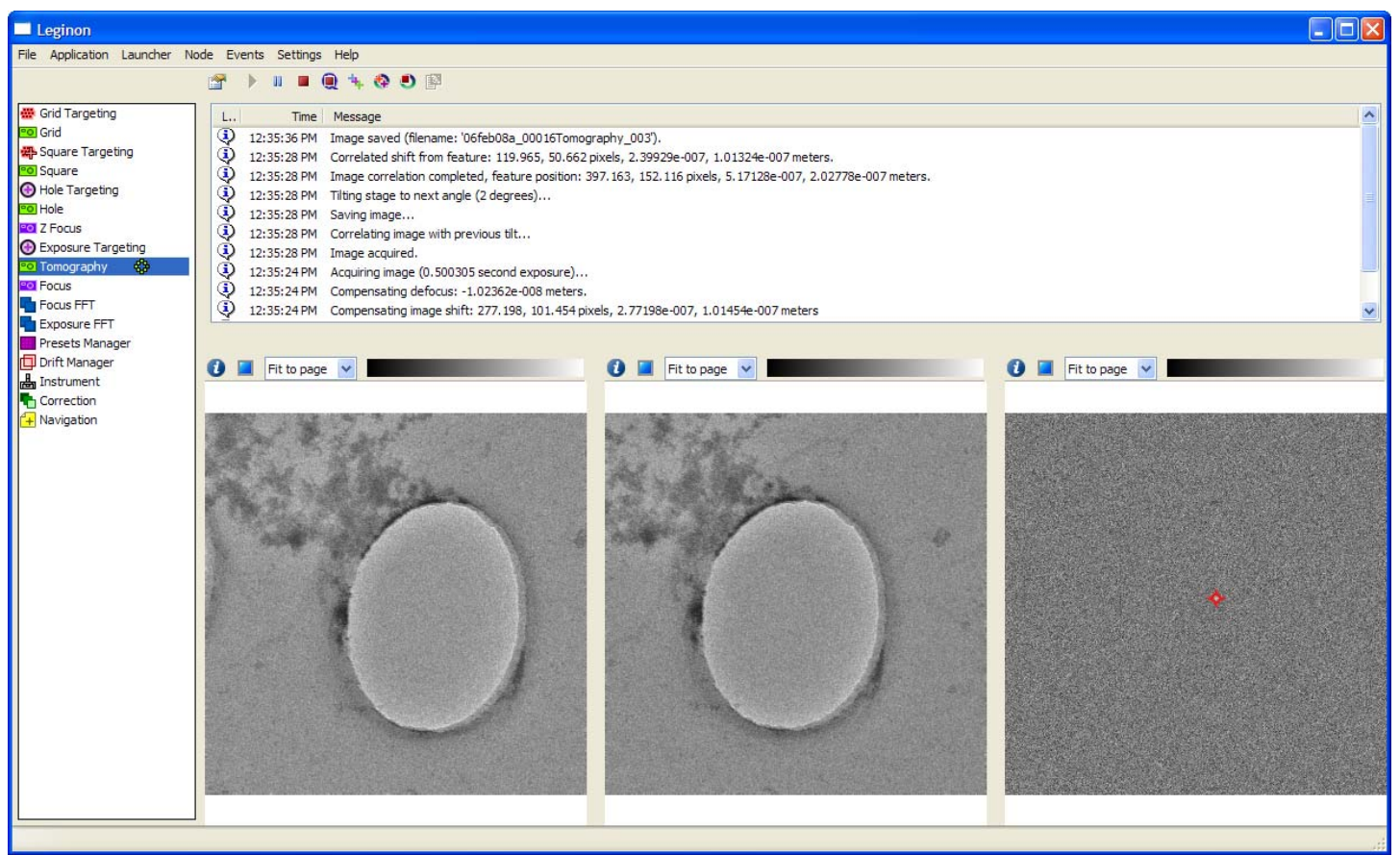

Fig. 1. Screenshot of Leginon collecting a tilt series.

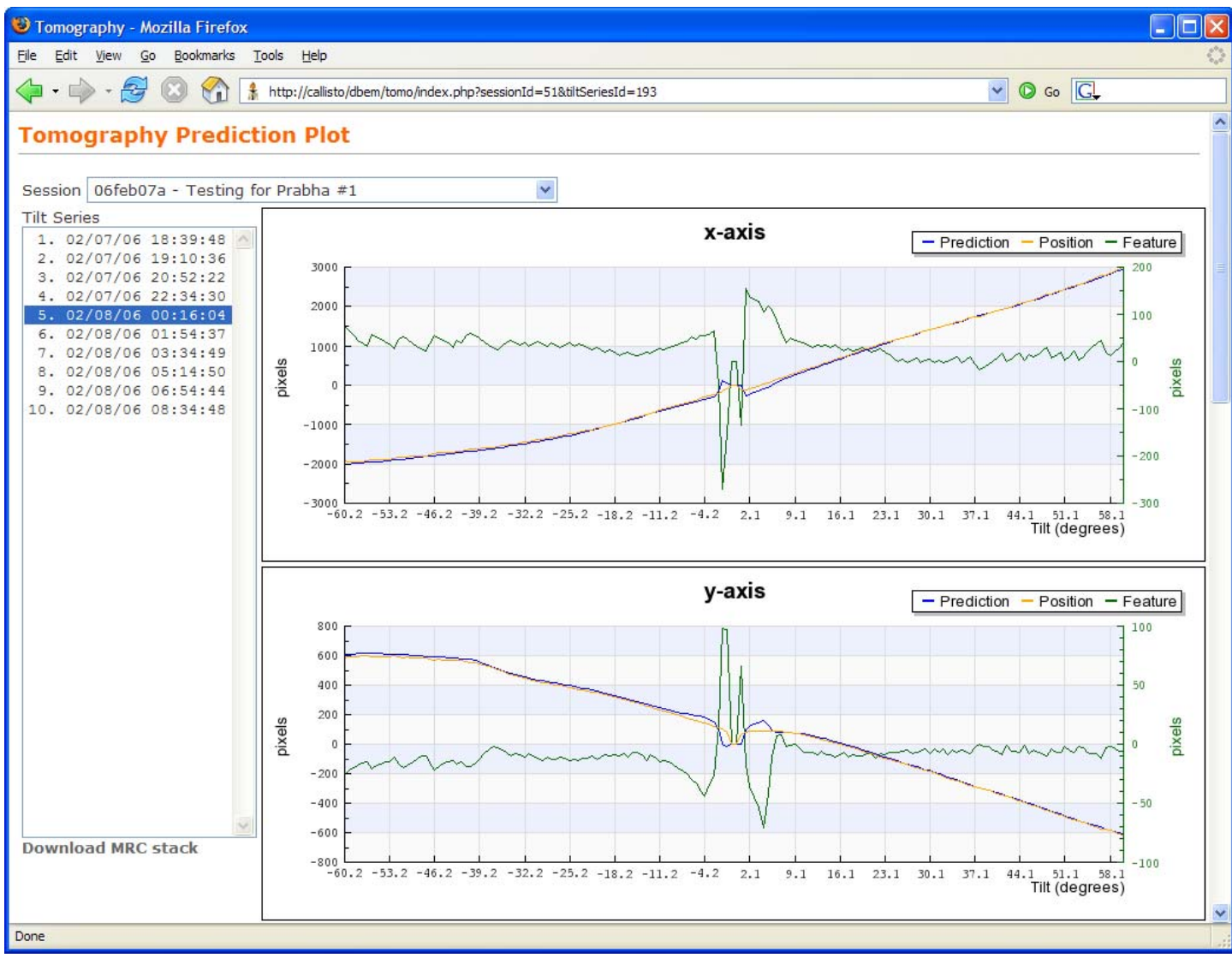

Fig. 2. Screenshot of tilt series diagnostic web tool. Graphs show the predicted (blue), actual (orange) and relative positions (green) of the tilt series in pixels per tilt angle in degrees for the $\mathrm{x}$ and $y$ axes of the CCD camera. 